\title{
Implementation of Fall Detection and Localized Caring System
}

\author{
Ming-Chih Chen, ${ }^{1}$ Yi-Wen Chiu, ${ }^{2}$ Chien-Hsing Chen, ${ }^{3}$ and Ei-Jo Chen ${ }^{1}$ \\ ${ }^{1}$ Department of Electronic Engineering, National Kaohsiung First University of Science and Technology, Kaohsiung City 811, Taiwan \\ ${ }^{2}$ Department of Social Work and Service Management, Tatung Institute of Commerce and Technology, Chiayi 612, Taiwan \\ ${ }^{3}$ Department of Information Technology, Meiho University, Pingtung 912, Taiwan
}

Correspondence should be addressed to Ming-Chih Chen; mjchen@nkfust.edu.tw

Received 13 September 2013; Accepted 12 October 2013

Academic Editor: Teen-Hang Meen

Copyright (C) 2013 Ming-Chih Chen et al. This is an open access article distributed under the Creative Commons Attribution License, which permits unrestricted use, distribution, and reproduction in any medium, provided the original work is properly cited.

We propose the fall detection and localized caring system to effectively detect the postures of a human and provide a service of remote connection with health care center. Especially, when elderly people fall down, they often need to be hospitalized. The system can inform the care center automatically through the wireless sensor network and send the rapid information of the incident for their family through the mobile phone. It also provides the location of incident for immediate rescue by dispatching medical staff from the center. The experimental results show that our system achieves the accurate rate of $99.9 \%$ for detecting a human fall and provides the care services effectively.

\section{Introduction}

According to the statistics in Taiwan, fall is the top second accidental cause of death happened in elderly people. The rate of falling down is about $15 \%$ to $40 \%$ every year, and the times of fall will increase along with their age. Most of the fall will cause the elderly people to be hospitalized or to reside in nursing home, and it will also cause the barriers of their daily activities. The risk factors of fall were found to be significantly associated with their osteoporosis disease, chronic disease, and so on. Even the slight fall will cause the most danger to elderly people.

The damage degree of fall for elderly people is often decided by the time of discovery, transport, and emergency medical service. The development of electronic technologies let the integration of sensors and wireless network become more and more popular. The automatic system management reduces the neglect from people and realizes the zerodistance medical treatment. A design deploys several infrared and temperature sensors in a room for detecting the fall of a human by receiving the information from sensors and performing detection algorithm [1].

More accelerometers and related circuits are used [2-12], and more costs are needed when the system is designed.
A portable device with two-axis accelerometer is worn on a shank for detecting the fall [2], and two similar designs use the same detection method but put the device on the waist $[3,4]$. In 2009, a special design puts two devices with three-axis accelerometers on the chest and thigh separately for observing the changes under different postures [5]. The fall detection method in [6] uses a barometric pressure sensor with an accelerometer to improve the recognition accuracy. A new design puts eight accelerometers in the washable pullover for detecting the motions of the torso and the upper body [8]. A hidden-Markov-model-(HMM-) based method is used to detect and predict a fall event [10]. The method in [11] uses two accelerometers and four surface electromyography sensors to effectively detect the human fall.

Our design uses only one accelerometer and achieves the fall detection with high accuracy. The system will be deployed in the long-term care institution. It can deliver the falling information through the connection of wireless sensor network (WSN), Ethernet, and Internet. The elderly people wear a fall detection device to detect the activities. If the fall situation is happened, the wearing device sends the falling message to the unit of medical treatment automatically. The design of such system can increase the living time of sufferers and reduce the rate of death due to the fall. 


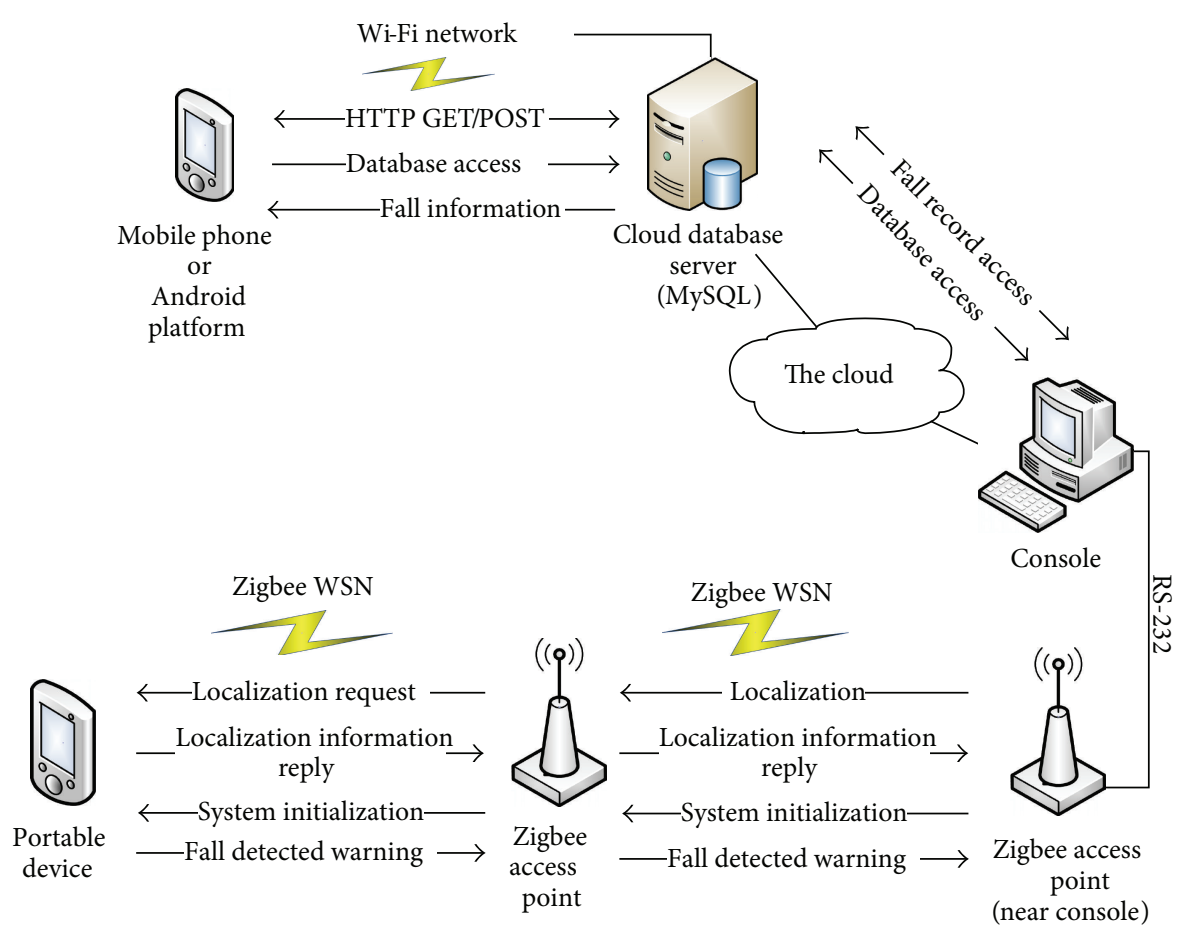

FIGURE 1: Proposed caring system architecture.

The implementation of our caring system includes hardware (HW) and software (SW) platforms that are described in Section 2. Sections 3 and 4 introduce the detailed HW and SW implementation, respectively. The experimental results are involved in Section 5, and the conclusion is given in Section 6.

\section{Proposed Caring System Architecture}

Our caring system architecture uses three kinds of networks including wireless sensor network, 802.11 wireless network (also called Wi-Fi), and Internet. As Figure 1 shows, the system architecture includes portable device, Zigbee access point, console, cloud database server, and mobile phone. The portable device in this system can detect the fall automatically and transmit the warning message to the nursing station through the Zigbee access point.

When a user wears the portable device but falls under the cover of wireless sensor network, the device automatically transmits the fall message to the console deployed in the nursing station. The station dispatches a nurse to find the user according to the indoor localization function and provide the required services according to the case history searched from the cloud database server. The system also informs the destined family of the user via mobile phone and shows the information of the rescue processes such as the time of fall, the arriving time of the nurse, the time of taking medical treatment, and so on.

Figure 1 also shows the communication information between two adjacent devices. For example, the portable device should communicate with Zigbee access point, especially the fall happens. The access point delivers the request of localization and system initialization from the console and the localization information and the warning of fall console to the console. The console also searches, records, and updates the case history in the database of MySQL.

The cloud sever performs three kinds of programs including the network connection program with the console and mobile phone and the users' database. When the system is utilized in a medium or small care institution, the cloud server could combine with the console and perform it in the nursing station. Figure 1 shows a caring network which utilizes cloud database server for managing a large number of institutions. One of the institutions connects the fall detection subsystem with the cloud database server through the console setup in the institution. The centralized database management can reduce the system costs effectively and manage the case history of users efficiently. Our caring system is suitable for each type of care institutions.

\section{Proposed System Hardware Platform}

In this system, there are two hardware designs including portable device worn by user and Zigbee access point deployed in the environment. The portable device is utilized to autodetect the fall of user as shown in Figure 2. It consists of a microcontroller unit (MCU), a three-axis accelerometer, a Zigbee WSN module, and a buzzer. The ARM9 MCU receives the analog signals from the accelerometer, performs the fall detection algorithm, and sends the warning message through Zigbee module if the user falls. The powers of all modules are supplied by a $1000 \mathrm{~mA}$ Polymer Lithium-Ion battery via a $5 \mathrm{~V}$ to $3.3 \mathrm{~V}$ voltage regulator. The battery has stable, slim, and extremely light characteristics which are suitable for 


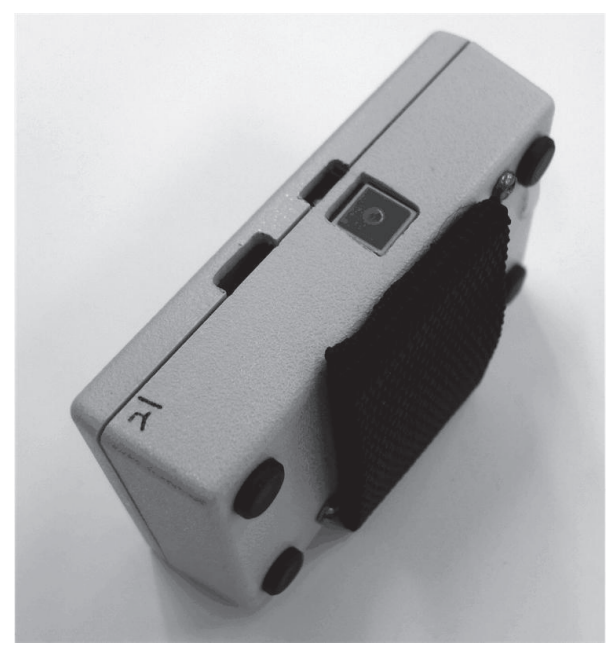

Figure 2: The portable device.

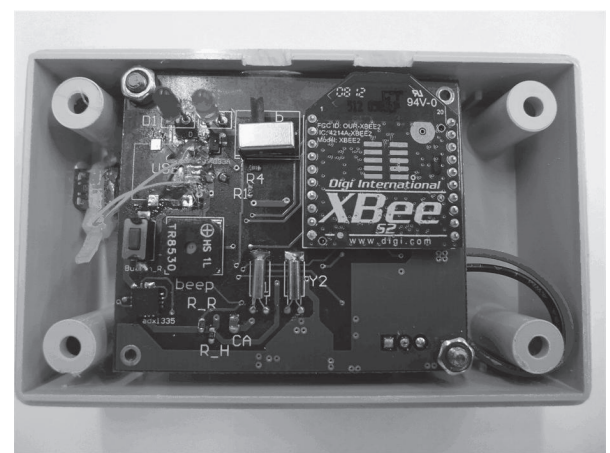

FIgURE 3: The circuit board of the Zigbee access point.

the portable device. The prototype of the portable device is embedded in a box with the dimensions of $8 \times 4 \times 2.5 \mathrm{~cm}^{3}$. The device can operate over one month on standby mode or continuously transmit fall information over 500 times in the experiment.

Figure 3 shows the circuit board of Zigbee access point. It consists of two modules including an ARM9 MCU and a Zigbee module. The Zigbee module is placed in the front of the board and is utilized to transfer the information between portable devices and the console. The Zigbee access point is deployed in the ceiling or upper side of the room and supplies the power by a wall adapter power supply with $5 \mathrm{~V}$ DC. The deployment of the access point needs to consider its signal attenuation in an environment with multicorner or shelter. The Zigbee access point connects with the console through the COM or USB port. The RS-232 communication protocol is performed between the console and the circuit board. The circuit board only uses Zigbee module for the wireless communication with other Zigbee access points, but it does not need a MCU to control the Zbigee. The console controls the Zigbee directly by sending commands through RS-232 protocol.

The appropriate deployment of access points in an institution contributes to the well performance of the localization function. The localization function can be performed by the triangulation method [13]. For example, the console sends the command to localize a user with a fixed portable device. The identification (ID) number of the portable device is known from the search of the database. The console broadcasts the request through all the access points. The portable device will receive the request from the near access points. The values of received signal strength indicator (RSSI) measured between the portable device and the near access points will be transmitted to the console. The console performs the triangulation method to calculate the distance between the nearest three access points. The estimated location of the user will be shown in the graphic interface of the caring system. From our experiment, we observe that the triangulation method can provide an approximate location in the visible range after adding the consideration of signal attenuation.

\section{Proposed System Software Platform}

Figure 4 shows the graphic user interface of the caring system. The interface is written by Borland $\mathrm{C}++$. The left upper side of the interface shows the map of interior space in the institution. When a user wears a portable device and comes into the cover area of the wireless sensor network, the location of the user will be shown in the map. The nurse can point to the location of the user, and the system can show the user's essential information including his or her photo, name, sex, birthday, and blood type in the right upper side. The bottom of the interface shows the serial number of user's device and the plane position of the user.

The nurse can also find the user's detailed information in the next page including his case history and emergency contact person. The system can set different authorities of each staff in the institution for protecting user's information and also record which staff uses the database, his login/logout time, and executed commands against the database.

The database records user's information in the cloud server or in the console depending on the selection from institution. The design of the database utilizes MySQL which is an open source relational database management system (RDBMS). The database provides multiuser access through wire, wireless, and $3 \mathrm{G}$ networks. It also allows the administrator to gather statistics and perform analyses by extracting their physiological information, care histories, the locations of fall, and so on. The danger zones in the institution for the residents also can be found and improved after analyzing the information of fall related.

\section{Experimental Results}

5.1. Experimental Results of Our Fall Detection Algorithm. The data flow of our fall detection algorithm is shown in Figure 5. How to detect the fall accurately is the most important issue in the caring system. There are five types of the fall happened when people fall including falling forward, falling backward, falling leftward, falling rightward, and falling slowly. The experiment selects fifty people to wear the portable device which performs the fall detection algorithm for verifying the accuracy of fall detection. They perform fifty times for each 


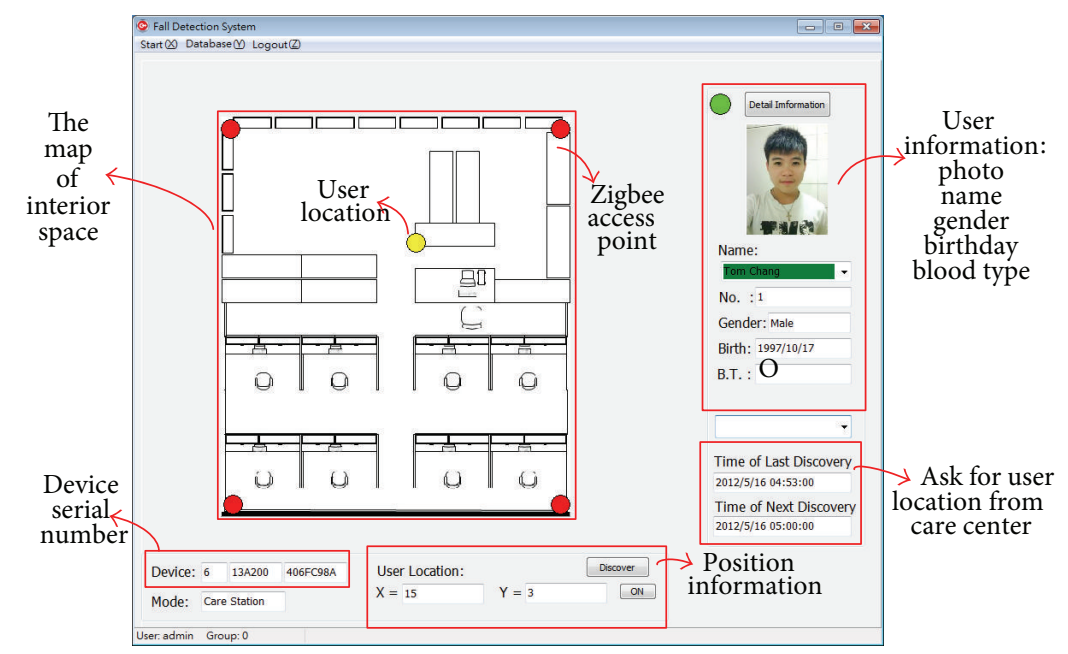

Figure 4: The user interface of caring system.

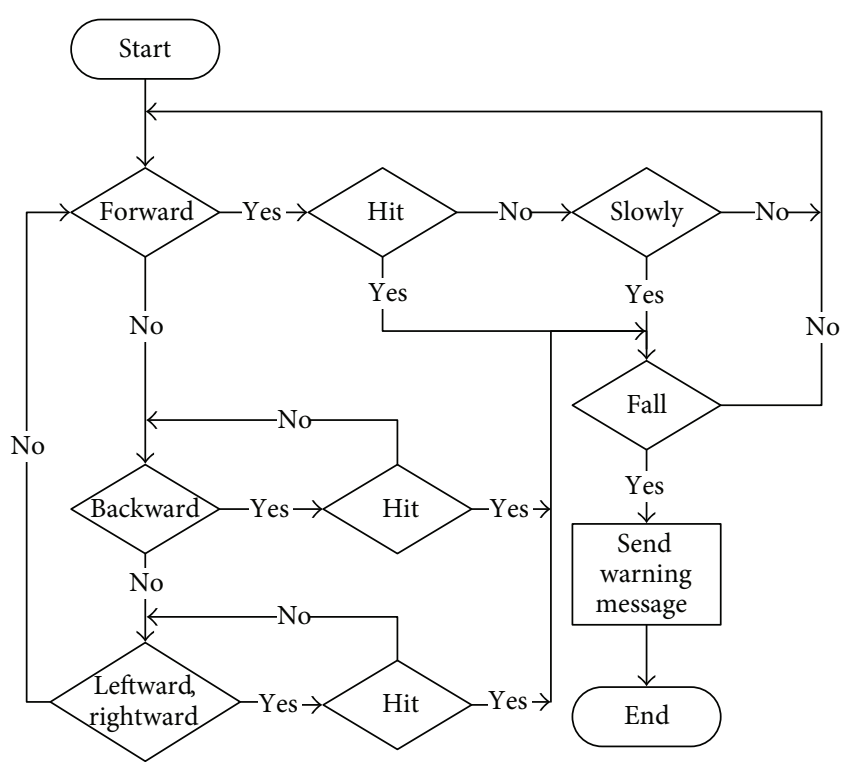

FIgURE 5: The fall detection algorithm.

fall type and the same times for each movement type. 12,500 times of falls and 12,500 times of movements are performed in the experiment.

Table 1 shows the experimental results by recording the hit or miss when a fall happened. We observe that the algorithm achieves the accurate rate of $100 \%$ in judging falling forward, falling leftward, and falling slowly. And the algorithm also achieves the accurate rate of $99.8 \%$ in judging falling backward, and the accurate rate of $99.9 \%$ in judging falling rightward. The algorithm also performs the judgment of different movements in Table 2. For example, there are five times of judgment misses in performing 2,500 times of jumps. The recognition accuracy of $100 \%$ is achieved in the judgments of running, going upstairs or downstairs, and lying down. For solving the miss of fall detection, a button is designed for emergency call on the portable device, and the caring system performs the indoor localization function per
TABLE 1: Experimental results of different fall types.

\begin{tabular}{lccccc}
\hline Fall type & Forward & Backward & Leftward & Rightward & Slowly \\
\hline Hit (times) & 2,500 & 2,495 & 2,500 & 2,498 & 2,500 \\
Miss (times) & 0 & 5 & 0 & 2 & 0 \\
Accuracy (\%) & $100 \%$ & $99.8 \%$ & $100 \%$ & $99.9 \%$ & $100 \%$ \\
\hline
\end{tabular}

ten minutes to monitor the location of the user. These two methods can deal with the miss of fall detection effectively in our experiment.

5.2. Performance Comparisons with Different Methods. This work also evaluates the performance of different fall detection methods by using three widely used criteria in fall detection systems. Two major criteria are adopted for performance comparisons in $[6,7,11]$ as shown in

$$
\begin{aligned}
& \text { sensitivity }(\%)=\frac{\mathrm{TP}}{\mathrm{TP}+\mathrm{FN}} \times 100 \%, \\
& \text { specificity }(\%)=\frac{\mathrm{TN}}{\mathrm{TN}+\mathrm{FP}} \times 100 \%,
\end{aligned}
$$

where the parameters TP, FP, FN, and TN defined in (1) can be expressed as shown in Table 3.

Parameter TP refers to a subject having fallen, and the detection system can detect it accurately; on the other hand, a system having failed to detect a fall of a subject is denoted by parameter FN. Parameter TN denotes a subject having experienced a false fall, and the system can recognize it correctly. An event in which a subject experiences a false fall yet the system recognizes it as a fall is denoted by parameter FP. These four parameters are used to calculate the times of the four possible recognition events. The criterion sensitivy refers to the correct rate of a system that can recognize the falls under all of the fall events. The criterion specificity denotes the correct rate of a system that can recognize the false falls under all of the false fall events. 
TABLE 2: Experimental results of special movements.

\begin{tabular}{lccccc}
\hline Movement type & Run & Jump & Go upstairs or downstairs & Lie down & Collide \\
\hline Hit (times) & 2,500 & 2,495 & 2,500 & 2,500 & 2,497 \\
Miss (times) & 0 & 5 & 0 & 0 & 3 \\
Accuracy (\%) & $100 \%$ & $99.8 \%$ & $100 \%$ & $100 \%$ & $99.9 \%$ \\
\hline
\end{tabular}

TABLE 3: Comparison of fall detection methods.

\begin{tabular}{|c|c|c|c|c|}
\hline Method & Bianchi et al. [6] & Aziz and Robinovitch [7] & Cheng et al. [11] & Ours \\
\hline \multicolumn{5}{|l|}{ Criterion } \\
\hline Recognition accuracy (\%) & 96.9 & 97 & 95 & 99.9 \\
\hline Sensitivity (\%) & 97.5 & 96 & 95.3 & 99.9 \\
\hline Specificity (\%) & 96.5 & 98 & 97.7 & 99.9 \\
\hline
\end{tabular}

Table 3 reveals that the proposed system has a sensitivity of $99.9 \%$ and a specificity of $99.9 \%$. This work has been compared with the methods of Bianchi et al. [6], Aziz and Robinovitch [7], and Cheng et al. [11], respectively. According to Table 3, the method in [7] has a higher recognition accuracy and specificity than those of the methods in $[6,11]$. Nevertheless, the proposed system performs better than the three previous designs.

\section{Conclusions}

In this paper, we design a localized caring system with the capability of fall detection. The fall detection algorithm proposed in the system can achieve an average accurate rate of $99.9 \%$ for detecting the fall down. The system allows residents in a care institution to wear a portable device which can reduce more damages from their falls due to the fall detection of high accuracy. The system also enables the localization function to help the nurse to find someone or take care of the residents. The design of cloud database server can record more information of the residents and help the administrator to perform analyses based on different classes of records. The system provides efficient and effective caring services for a care institution.

\section{Acknowledgment}

This work is supported in part by Taiwan's Ministry of Education under Project no. 101B-09-027.

\section{References}

[1] A. Sixsmith and N. Johnson, "A smart sensor to detect the falls of the elderly," IEEE Pervasive Computing, vol. 3, no. 2, pp. 4247, 2004.

[2] K. T. Song and Y. Q. Wang, "Remote activity monitoring of the elderly using a two-axis accelerometer," in Proceedings of the CACS Automatic Control Conference, pp. 1-6, 2005.

[3] T. Zhang, J. Wang, L. Xu, and P. Liu, "Fall detection by wearable sensor and one-class SVM algorithm," Lecture Notes in Control and Information Sciences, vol. 345, pp. 858-863, 2006.

[4] C. Doukas, I. Maglogiannis, P. Tragas, D. Liapis, and G. Yovanof, "Patient fall detection using support Vector Machines," IFIP
International Federation for Information Processing, vol. 247, pp. 147-156, 2007.

[5] Q. Li, J. A. Stankovic, M. A. Hanson, A. T. Barth, J. Lach, and G. Zhou, "Accurate, fast fall detection using gyroscopes and accelerometer-derived posture information," in Proceedings of the 6th International Workshop on Wearable and Implantable Body Sensor Networks (BSN '09), pp. 138-143, June 2009.

[6] F. Bianchi, S. J. Redmond, M. R. Narayanan, S. Cerutti, and N. H. Lovell, "Barometric pressure and triaxial accelerometry-based falls event detection," IEEE Transactions on Neural Systems and Rehabilitation Engineering, vol. 18, no. 6, pp. 619-627, 2010.

[7] O. Aziz and S. N. Robinovitch, "An analysis of the accuracy of wearable sensors for classifying the causes of falls in humans," IEEE Transactions on Neural Systems and Rehabilitation Engineering, vol. 19, no. 6, pp. 670-676, 2011.

[8] G. Yongli, O. S. Yin, and P. Y. Han, "State of the art: a study on fall detection," World Academy Science, Engineering and Technology, vol. 62, pp. 294-298, 2012.

[9] Y. W. Bai, S. C. Wu, and C. L. Tsai, "Design and implementation of a fall monitor system by using a 3-axis accelerometer in a smart phone," IEEE Transactions on Consumer Electronics, vol. 58, no. 4, pp. 1269-1275, 2012.

[10] L. Tong, Q. Song, Y. Ge, and M. Liu, "HMM-based human fall detection and prediction method using tri-axial accelerometer," IEEE Sensors Journal, vol. 13, no. 5, pp. 1849-1856, 2013.

[11] J. Cheng, X. Chen, and M. Shen, "A framework for daily activity monitoring and fall detection based on surface electromyography and accelerometer signals," IEEE Journal of Biomedical and Health Informatics, vol. 17, no. 1, pp. 38-45, 2013.

[12] W. C. Cheng and D. M. Jhan, "Triaxial accelerometer-based fall detection method using a self-constructing cascade-AdaBoostSVM classifie," IEEE Journal of Biomedical and Health Informatics, vol. 17, no. 2, pp. 411-419, 2013.

[13] C. C. Pu, Development of a new collaborative ranging algorithm for RSSI indoor location tracking in WSN [Ph.D. thesis], Dongseo University, Busan, South Korea, 2009. 


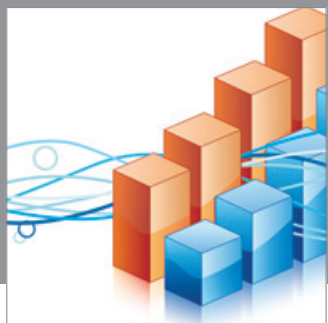

Advances in

Operations Research

mansans

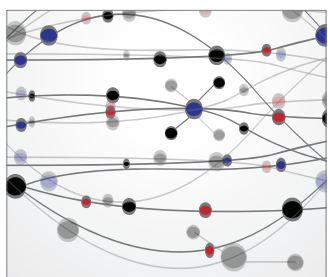

The Scientific World Journal
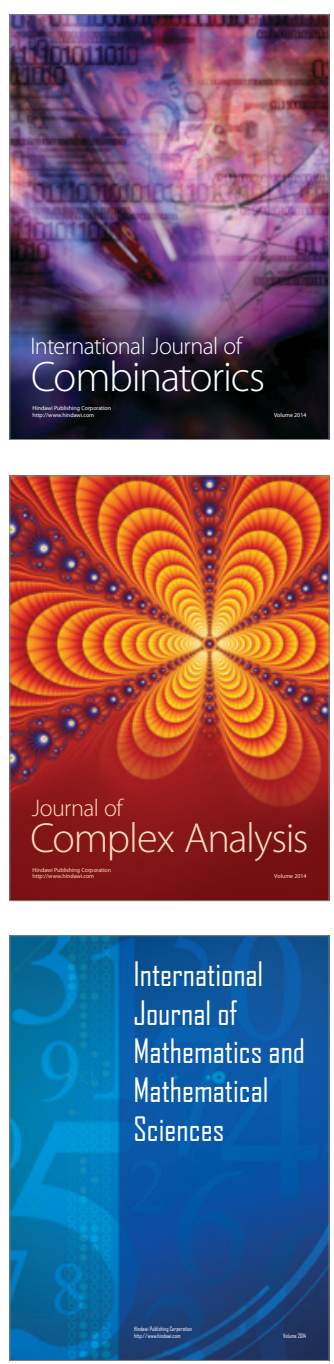
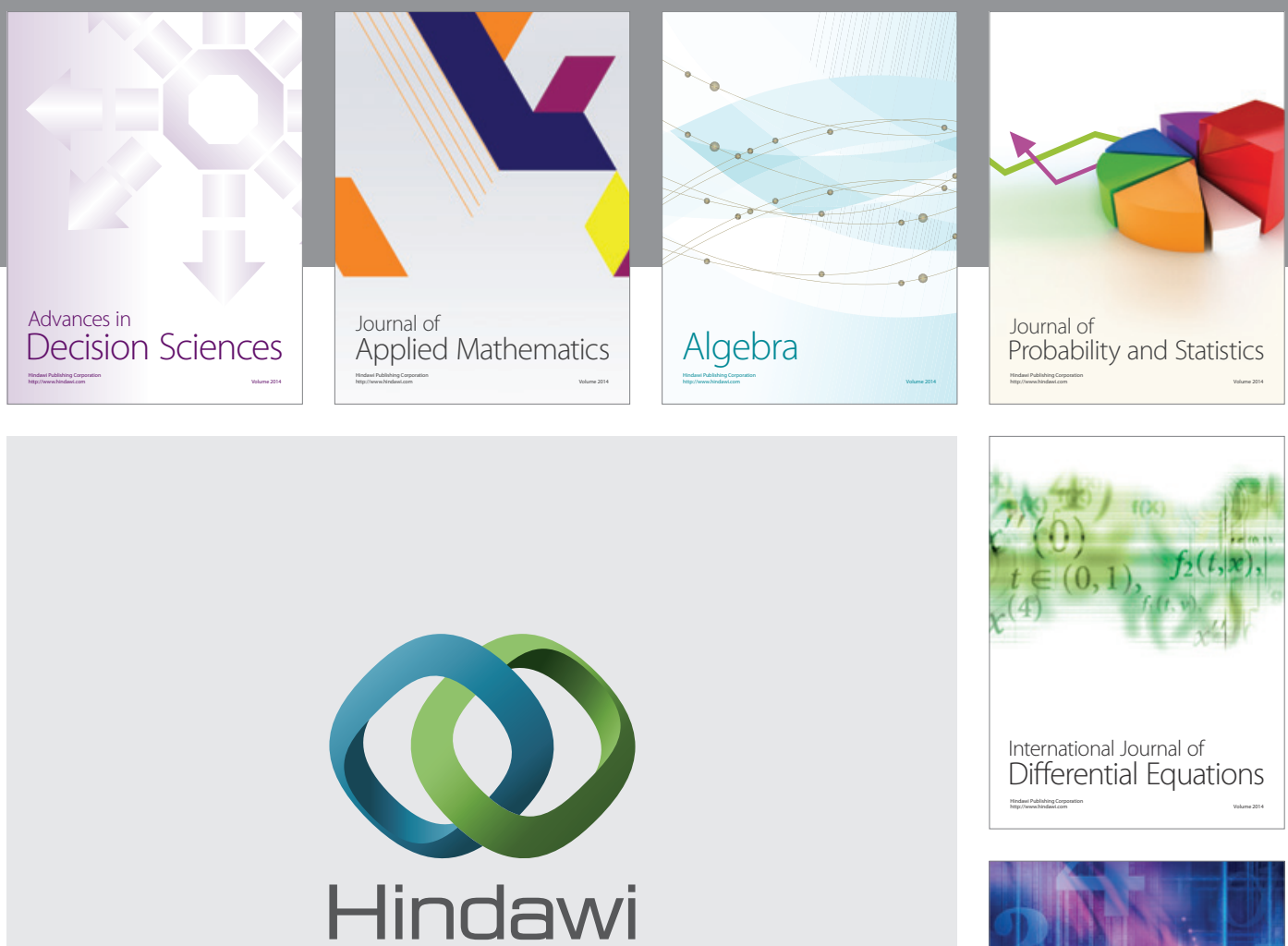

Submit your manuscripts at http://www.hindawi.com
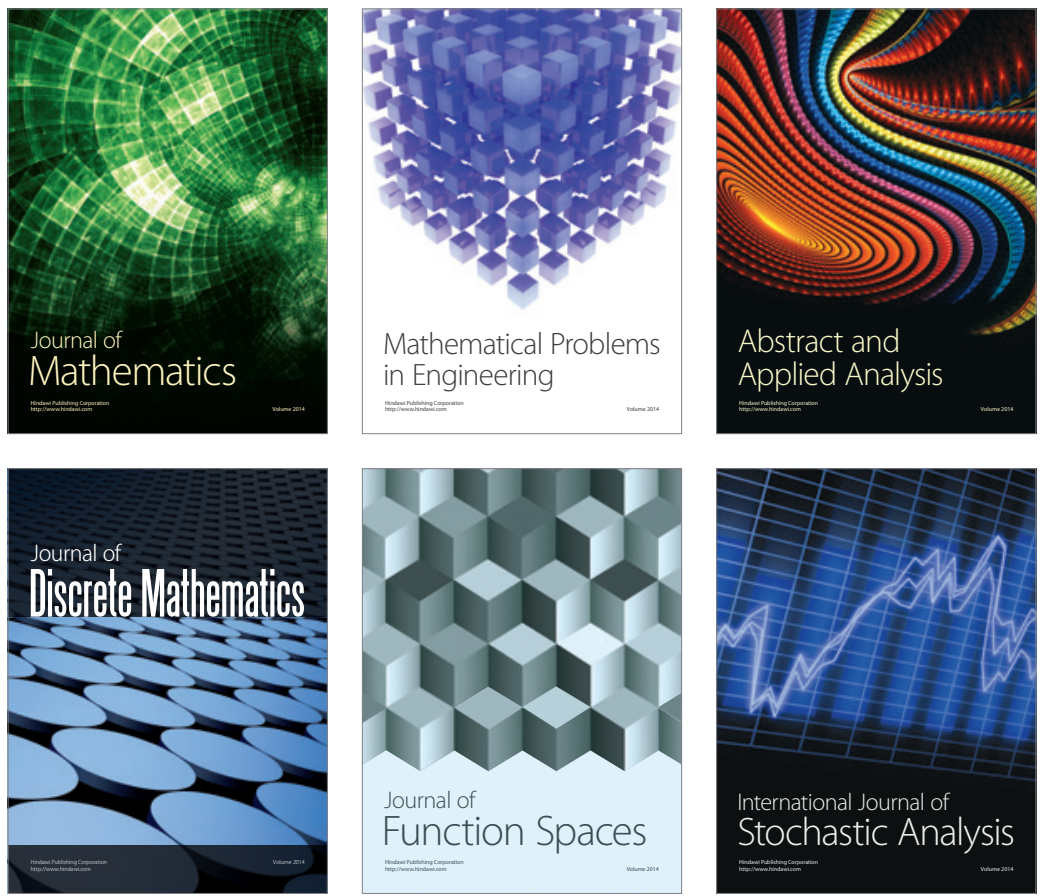

Journal of

Function Spaces

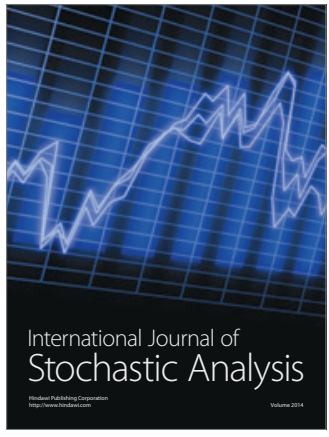

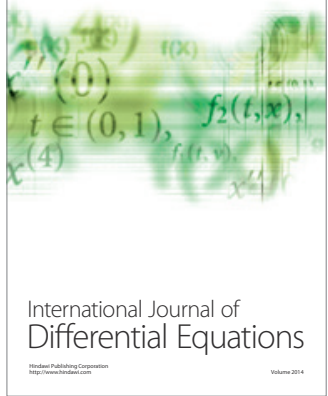
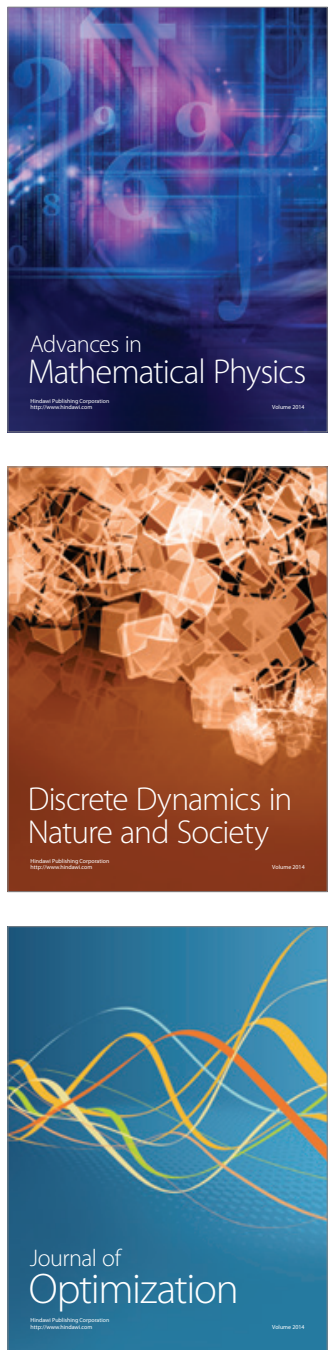